P.O. Box 10084

(312) 326-5007

Chicago, IL

60610-0084

8 June 1993

"HUMAN GENOME SEQUENCING WITH DIRECT

X-RAY HOLOGRAPHIC IMAGING"

FINAL REPORT

DoE Grant \#DE-FG02-89ER60898

Prepared by: $\quad$ Dr. Charles K. Rhodes

President

MCR Technology Corporation

P.O. Box 10084

Chicago, IL 60610-0084

Prepared for: $\quad$ Dr. Gerald Goldstein

U.S. Department of Energy

ER-74 Germantown

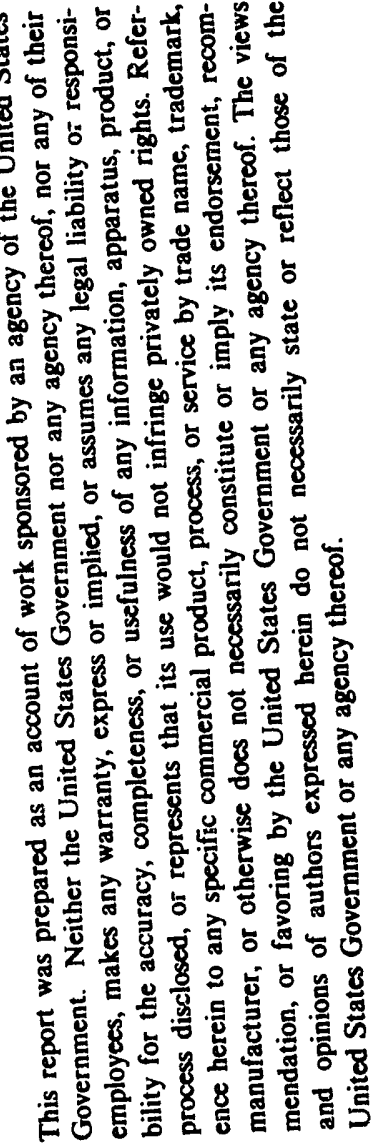

Washington, D.C. 20545 


\section{CONTENTS}

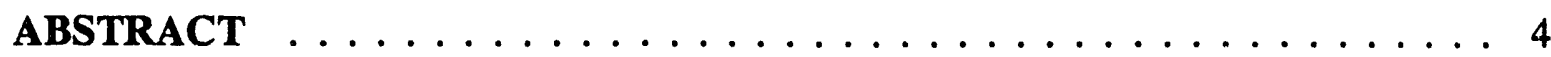

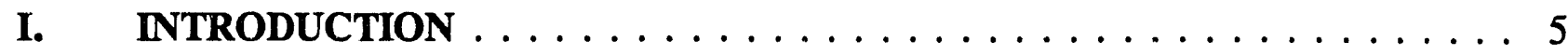

II. DISCUSSION OF RESEARCH AND DEVELOPMENT $\ldots \ldots \ldots \ldots \ldots$. . 5

A. HOLOGRAPHIC CAMERA ...............5

B. CHEMISTRY FOR TAGGED BASE AND ENZYMOLOGY FOR SAMPLE PREPARATION $\ldots \ldots \ldots \ldots \ldots \ldots$

C. X-RAY LIGHT SOURCE TECHNOLOGY $\ldots \ldots \ldots \ldots \ldots \ldots$

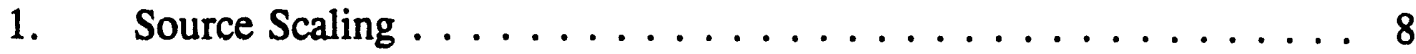

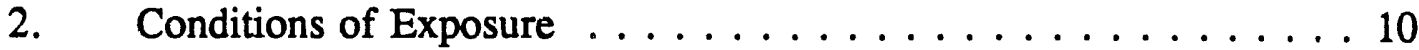

a. Bleaching of the Tags ...............

b. Image Blurring . . . . . . . . . . . . . . . . 13

c. Influence of Reference Scattering Sphere on Image Quality ..................... 16

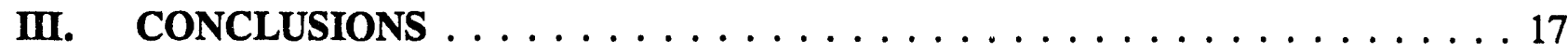

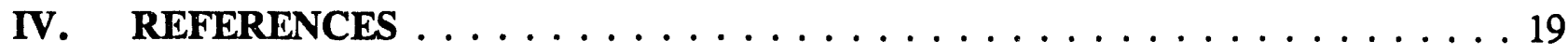

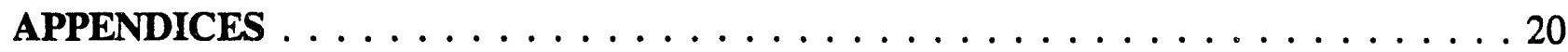

APPENDIX A: "Genome Sequencing by Direct Imaging X-Ray Color Holography" . . . . . . . . . . . . . . . . . . 20

APPENDIX B: "Human Genome Sequencing with Direct X-Ray Holographic Imaging", Steroids, Ltd. Final Technical Report . . . . . . . . . 25

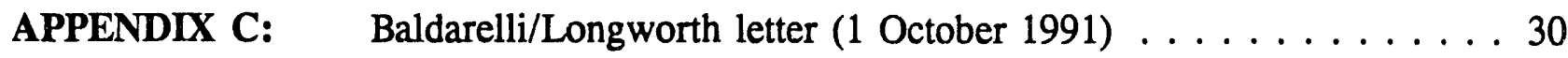

APPENDIX D: "Method of Concentration of Power in Materials for X-Ray Amplification" . .................. 36

APPENDIX E: "Observation of Relativistic and Charge-Displacement SelfChanneling of Intense Subpicosecond Ultraviolet (248 $\mathrm{nm})$ Radiation in Plasmas" ..................41

APPENDIX F: $\quad$ "Relativistic and Charge-Displacement Self-Channeling of Intense Ultrashort Laser Pulses in Plasmas" . . . . . . . . . . . . 45

APPENDIX G: "Stability Analysis of Relativistic and Charge-Displacement SelfChanneiing of Intense Laser Pulses" . . . . . . . . . . . . . 61 
APPENDIX H: "X-Ray Amplifier Energy Deposition Scaling with Channeled Propagation" ..................... 65

Reprints + Preprints removed conf papen cycled syaratily

3 


\begin{abstract}
Direct holographic imaging of biological materials is widely applicable to the study of the structure, properties and action of genetic material. A particular application involves the sequencing of the human genome. This new prospective genomic imaging technology is composed of three subtechnologies. They are (1) an x-ray holographic camera, (2) suitable chemistry and enzymology for the preparation of tagged DNA samples, and (3) the illuminator in the form of an $\mathrm{x}$-ray laser. The results of the research conducted under the support of this grant lead to three general conclusions concerning this emerging technology. They are $(\alpha)$ that an appropriate $\mathrm{x}$-ray camera, embodied by the instrument developed by MCR, is available and $(\beta)$ that suitable chemical and enzymatic procedures exist for the preparation of the necessary tagged DNA strands. The third conclusion $(\gamma)$ concerns the future development of the $x$-ray illuminator. The finding here, which is anchored on the results of research in several areas, stemming directly from work conducted by the principals of MCR, is that a practical small scale $\mathrm{x}$-ray light source is indeed feasible. This outcome requires the use of unconventional physical processes in order to achieve the necessary power-compression in the amplifying medium. The understanding of these new physical mechanisms is developing rapidly. Importantly, although the $\mathrm{x}$-ray source does not currently exist, the understanding of these new physical mechanisms is developing rapidly and the research has established the basic scaling laws that will determine the properties of the $\mathrm{x}$-ray illuminator. When this $\mathrm{x}$-ray source becomes available, an extremely rapid and cost effective instrument for 3-D imaging of biological materials can be applied to a wide range of biological structural assays, including the base-pair sequencing of the human genome and many questions regarding its higher levels of organization.
\end{abstract}




\section{INTRODUCTION}

Holographic imaging as a general method is applicable to the visualization of biological materials over a wide range of electromagnetic wavelengths spanning, at least, from the visible to the $\mathrm{x}$-ray region. In particular, given sufficient contrast and the spatial resolving power potentially available with $\mathrm{x}$-rays, this raises the possibility for direct visualization of the structure of DNA. In principle, this could include the base-pair sequence analysis in addition to rotatable three-dimensional (3-D) images which would reveal the higher levels of DNA organization in chromatin and chromosomes. Therefore, an $\mathbf{x}$-ray holographic camera has been developed as the technology needed to directly image the human genome in order to achieve rapid sequencing. The basic idea of this approach is described in Appendix A.

The combination of three technologies is needed to form the required images of the genetic material for the base-pair sequencing. They are (1) a holographic camera suitable for operation in the $\mathrm{x}$-ray range, (2) appropriate chemical procedures and enzymology for producing tagged bases and DNA strands, and (3) a sufficiently bright source of radiation in the $\mathrm{x}$-ray range to serve as the illuminator. In this report we will discuss the progress made in these three areas.

\section{DISCUSSION OF RESEARCH AND DEVELOPMENT}

\section{A. HOLOGRAPHIC CAMERA}

Holographic $\mathrm{x}$-ray imaging of biological materials opens the door to an entirely new means of the visualization of living biological entities, including the structure of DNA. Significantly, this technology, because of its very high speed $\left(\sim 10^{-13} \mathrm{~s}\right)$ and spatial resolving power, will be able to view important features of biological structures that cannot be made by 
any other technique, either in current practice or proposed. Although not strictly required for DNA sequencing, the camera that has been developed by the Company is a holographic instrument and, consequentiy, is capable of recording full three-dimensional information in a single brief exposure. Experimental tests of the principles of its operation on histopathological specimens with visible radiation $(514.5 \mathrm{~nm}$ ) have recently demonstrated that the patented concept underlying the design of this instrument can achieve both high contrast and diffraction-limited spatial resolution in all three dimensions. Since the principles of operation are unchanged with the use of radiation in the $\mathrm{x}$-ray range, we conclude that this same high level of performance will also be characteristic of its function in that short wavelength region. We emphasize that, since this image is formed from information taken on a single exposure, an absolute requirement in the $\mathrm{x}$-ray range for the preservation of the structure of hydrated material, it is superior to any other known method of three-dimensional (3-D) imaging.

The basic instrument and its characteristics have been described elsewhere. ${ }^{1}$ In terms of spatial resolution with illumination at $514.5 \mathrm{~nm}$, the results are as follows: transverse, $1.40 \mu \mathrm{m}$ measured, $1.29 \mu \mathrm{m}$ diffraction limit; longitudinal, $10.0 \mu \mathrm{m}$ measured, $9.7 \mu \mathrm{m}$ diffraction limit. An example of the contrast achieved is shown in Fig. (1); all three dimensions exhibit sharp contrast. We emphasize that this result on Parascaris univalens embodies both high resolution and exceptional contrast. The complex structure observed is believed to be associated with the distribution of chromatin in the cell. A rupture in the nuclear membrane is clearly visible in Fig. (1) near the center of the image. We also note that a holographic technique, because it involves interference, can always be adjusted to give high contrast, independent of the scattering strength of the sample, hence, without the need for staining, by proper adjustment 
of the amplitude of the reference wave. Normal microscopic illumination does not have this property. Indeed, the sample shown in Fig. (1), when observed with a high quality biological microscope, exhibited quite poor contrast and the visibility of the features, although perceivable, was far less than that exhibited in Fig. (1).

Comparison of the results shown in Fig. (1), which were recorded on a single exposure, with images of chromosomes at mitosis, obtained with confocal scanning microscopy ${ }^{2}$, is informative. The holographic method exhibits essentially the same level of contrast, spatial resolution, and structural detail. Of course, the high damage rate to biological materials arising from $x$-ray exposure rules out the confocal approach in that spectral range. In the $x$-ray region, the single-pulse holographic approach is the only method that can produce $3-\mathrm{D}$ information, since it avoids the radiological damage problem.
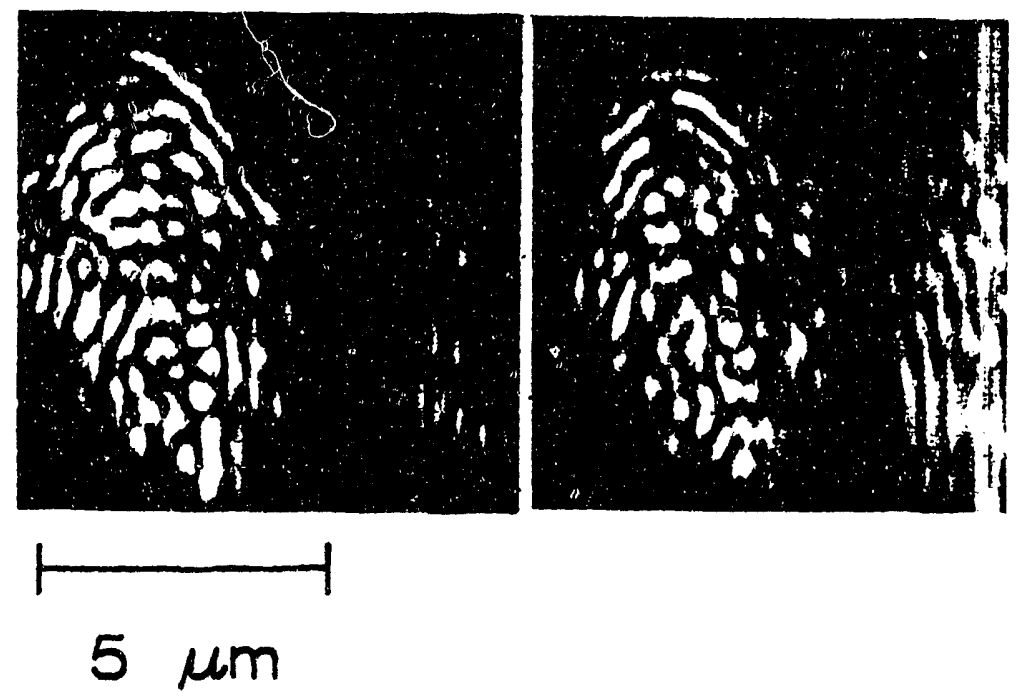

Figure (1): Larval Parascaris univalens interphase cell nucleus. Tomogram spacing $=7.2$ $\mu \mathrm{m}$. Both high contrast and diffraction limited spatial resolution were achieved. The complex structure observed is associated with the distribution of chromatin in the cell. 
The development and test of the holographic carnera has experimentally established the principles of operation that would apply to the $\mathrm{x}$-ray range. An $\mathrm{x}$-ray version of the camera has already been constructed. Given the availability of an appropriate $\mathrm{x}$-ray source, this imaging technology could readily serve the requirements needed for genome sequencing as described in Appendix A.

B. CHEMISTRY FOR TAGGED BASE AND ENZYMOLOGY FOR SAMPLE PREPARATION

Steroids, Ltd., under a subcontract on this program, has synthesized several halogentagged bases. A report of their results is contained in Appendix B. The conclusion is that the required synthesis can be performed.

Tests of the incorporation of some of the synthesized tagged bases was also investigated. These experiments were conducted by Dr. James Baldarelli at Harvard. A letter to Dr. Longworth (1 October 1991) describing these results appears in Appendix C. On the basis of these findings, we do not expect the enzymology to prevent the attainment of the goals of this program.

\section{X-RAY LIGHT SOURCE TECHNOLOGY}

\section{Source Scaling}

$\mathrm{X}$-ray microholography performed with a single pulse requires an $\mathrm{x}$-ray source with the exceptionally high brightness figure characteristic of an $\mathrm{x}$-ray laser. The critical governir ; issue, which determines the scaling relationship between the required excitation energy $(E):$ id the amplifier gain $(G)$ of $x$-ray lasers, is the spatial control of the deposited energy. Anal sis shows, as described in Appendix D, that optimizing the gain (G) per unit energy (E) call: for a guided mode of propagation in order to optimally control the deposition of the en rgy. 
Overall, in comparison to traditional forms of excitation, for a fixed x-ray energy output $\left(E_{x}\right)$ and wavelength $\left(\lambda_{x}\right)$, a reduction of several orders of magnitude in the necessary energy (E) results, if this form of confined (channeled) propagation can be achieved.

Recent experiments ${ }^{3}$, which are supported by carefully developed theoretical analysis ${ }^{4}$, have demonstrated the basic physics of a new form of self-channeled propagation exactly of the type necessary for the implementation of $\mathbf{x}$-ray lasers. These two developments are described in Appendices $\mathrm{E}$ and $\mathrm{F}$. These results represent a profound change in the field of $\mathrm{x}$-ray laser research because they alter drastically the fundamental scaling relationships among the relevant physical variables.

The stability of the self-channeled propagation is of fundamental importance for practical application of this phenomenon to x-ray amplification. A study of this question, as described in Appendix G, has shown that a large zone of stability exists that is compatible with strong $\mathrm{x}$ ray amplification.

The implications of this development for $\mathrm{x}$-ray microholography are extremely important and propitious. They are (1) that a properly controlled energy deposition rate, sufficient for the production of stimulated $x$-ray sources from the water window $\left(\hbar \omega_{x} \sim 400 \mathrm{eV}\right)$ up to a few kilovolts in quantum energy, can now be achieved, (2) that an x-ray output energy of $\sim 1 \mathrm{~mJ}$ per pulse is achievable with laboratory-scale technology, and (3) that an X-ray beam diamet ( $\sim \mu \mathrm{m}$ ), ideal for microholography in the Fourier configuration, arises as a nat $\downarrow$ consequence of the physics. Importantly, an x-ray source with these parameters is perf tly matched to the holographic requirements for biological materials necessary for the gen mic analysis in terms of all its relevant properties, specifically, wavelength ( $2-40 \AA)$, pulse e ergy 
$(\sim 1 \mathrm{~mJ})$, pulse length $\left(\sim 10^{-13} \mathrm{~s}\right)$, beam diameter $(\sim 1 \mu \mathrm{m})$, and divergence $(\sim 1 \mathrm{mrad})$.

\section{Conditions of Exposure}

The use of an $\mathrm{x}$-ray laser for biological imaging involves an extremely high exposure rate of both the specimen and the reference sphere. In this section we address some basic aspects of these questions. They are (1) the possibility that the tags may bleach owing to the exceedingly high intensity of exposure, (2) the blurring of the image that may occur owing to plasma motion, and (3) the influence of the high intensity on the optical properties of the reference scattering sphere. This analysis shows that the conditions of exposure required with an $\mathrm{x}$-ray source are consistent with the formation of a high-contrast, high-spatial-resolution image of tagged DNA.

a. Bleaching of the Tags

In the process of scattering, electrons from the $\mathrm{K}$ or $\mathrm{L}$ shell are expelled from the atom. Since the vacancy must be refilled in order for the atom to be used for subsequent scattering, we need to estimate how many times the atom can be recycled during the laser pulse. For present purposes we will consider the pulse length to be $\sim 100 \mathrm{fs}$.

The K-level line widths are well approximated ${ }^{5}$ by the expression

$$
\Gamma(K)=1.73 \times Z^{3.93} \times 10^{-6}(\mathrm{eV}) .
$$

Thus, the lifetimes of these states are given by

$$
\tau(K)=\frac{\hbar}{\Gamma(K)}=3.8 \times Z^{-3.93} \times 10^{-10} s .
$$


For the K-shell tags discussed in Appendix A, the values given in Table I apply.

\begin{tabular}{||c|c|c|c|c||}
\hline $\begin{array}{c}\text { Atom } \\
\text { (Edge) }\end{array}$ & $\begin{array}{c}\text { Quantum } \\
\text { Energy (Ev) }\end{array}$ & Wavelength $(\AA)$ & $\begin{array}{c}\text { Lifetime } \\
(\mathrm{fs})\end{array}$ & $\begin{array}{c}\text { Number of } \\
\text { Scatterings }\end{array}$ \\
\hline $\mathrm{F}(\mathrm{K})$ & 700 & 17.7 & 67.6 & 1.47 \\
\hline $\mathrm{Si}(\mathrm{K})$ & 1560 & 7.9 & 11.9 & 8.4 \\
\hline $\mathrm{S}(\mathrm{K})$ & 2472 & 5.0 & 7.05 & 14.1 \\
\hline $\mathrm{Cl}(\mathrm{K})$ & 2822 & 4.4 & 5.55 & 18.0 \\
\hline
\end{tabular}

Table I. Candidate DNA tagging atoms using the K-edge.

The last column of Table I shows how many times the K-shell electron can be reused during the laser pulse. A fluorine atom could only be used once, so to be a useful tag, many atoms would have to be attached to the base. Fluorine is only a marginally effective tag and has been rejected since its wavelength is somewhat longer than needed for the genome sequencing.

For L-shell tags, a simple expression for the widths is not available. However, from published line widths ${ }^{5}$ we can calculate the corresponding lifetimes for the candidate tags. These are displayed in Table II. 


\begin{tabular}{||c|c|c|c|c||}
\hline $\begin{array}{c}\text { Atom } \\
(\mathrm{Edge})\end{array}$ & $\begin{array}{c}\text { Quantum } \\
\text { Energy }(\mathrm{eV})\end{array}$ & $\begin{array}{c}\text { Wavelength } \\
(\AA)\end{array}$ & $\begin{array}{c}\text { Lifetime } \\
(\mathrm{fs})\end{array}$ & $\begin{array}{c}\text { Number of } \\
\text { Scatterings }\end{array}$ \\
\hline $\mathrm{Br}\left(\mathrm{L}_{1}\right)$ & 1782 & 7.0 & 0.0823 & 1220 \\
\hline $\mathrm{I}\left(\mathrm{L}_{1}\right)$ & 5188 & 2.4 & 0.188 & 531 \\
\hline $\mathrm{I}\left(\mathrm{L}_{3}\right)$ & 4557 & 2.7 & 0.227 & 441 \\
\hline $\mathrm{Se}\left(\mathrm{L}_{1}\right)$ & 1653 & 7.5 & 0.0877 & 1140 \\
\hline
\end{tabular}

Table II. Candidate DNA tagging atoms using L-edges.

To the original list of L-shell tagging atoms, we have added selenium, which has favorable biochemical properties for this purpose. All of the L-shell tags can be reused hundreds of times during the laser pulse.

It can be concluded that bleaching does not present a limitation, if we (a) tag with sufficiently high $\mathrm{Z}$ atoms, and (b) it is superior to use $\mathrm{L}$-shell electrons than $\mathrm{K}$-shell electrons.

The wavelength zone corresponding to the use of the tags indicated in Tables I and II are shown on the gain scaling curve in Fig. (2). It is significant that these wavelengths, as described in Appendix (H) can be produced with a laser technology having a pulse energy less than 1 Joule. This implies that a relatively small-scale solid state laser technology can be built. 


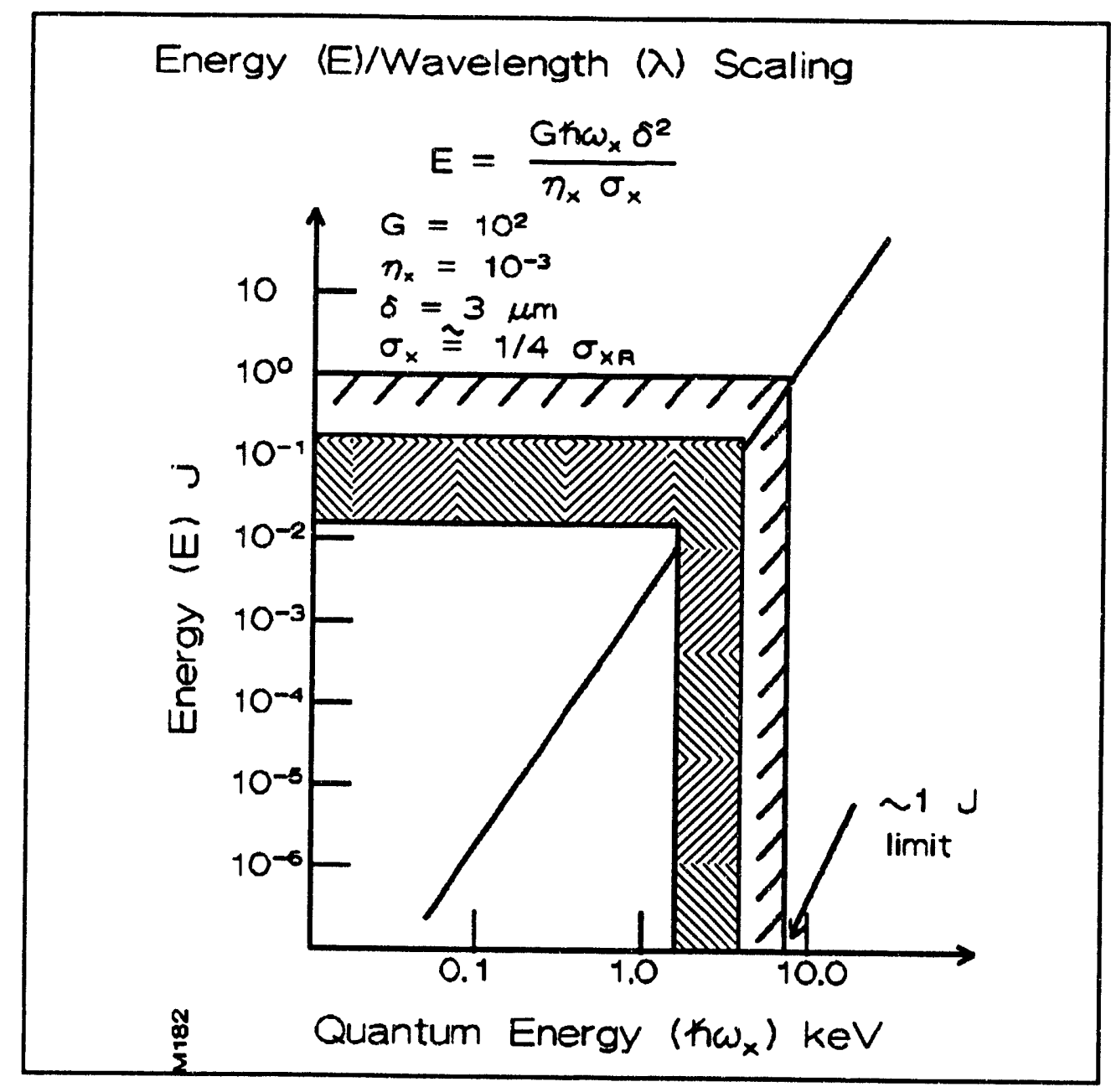

Fig. (2): Scaling relationship between required excitation energy (E) and quantum energy $\left(\hbar \omega_{x}\right)$ characteristic of the amplifier. Parameters: total gain exponent $\mathrm{G}=100$, energy efficiency $\eta_{\mathrm{x}}=10^{-3}$, channel diameter $\delta=3 \mu \mathrm{m}, \mathrm{x}$-ray $\left(\hbar \omega_{x}\right)$ cross section for stimulated emission $\sigma_{x}$, $x$-ray cross section for stimulated emission for radiatively-broadenet transition $\sigma_{\mathbf{x R}}$. The wavelength region corresponding to the genome sequencing is shown to involve a technology with a pulse energy less than one joule. See Appendix E for further discussion.

\section{b. Image Blurring}

The motion of tagging atoms during the pulse is a potential source of blurring in the hologram. We now estimate the magnitude of the expected blurring. 
Laser energy is initially deposits $d$ in the atomic electrons far out of thermal equilibrium, primarily in the form of $\sim 2-\mathrm{keV}$ electrons. The collision time is a good measure of the time it takes for the electron energy distribution to reach thermal equilibrium. Spitzer ${ }^{6}$ has shown that

$$
t_{c}=\frac{m^{1 / 2}(3 k T)^{3 / 2}}{8 \times 0.714 \pi n e^{4} Z^{4} \ln \Lambda}=0.266 \frac{T^{3 / 2}}{n \ln \Lambda}(s),
$$

where $n$ is the electron density and $\ln \Lambda$ is the Coulomb logarithm.

Since typical conditions of exposure would involve $\sim 1.5 \times 10^{12}$ photons with a wavelength of $6.07 \AA$ on a carbon foil with an area of about $9 \times 10^{-8} \mathrm{~cm}^{2}$. The total energy flux is then $3.4 \times 10^{22} \mathrm{Ev} \cdot \mathrm{cm}^{-2}$ or $5.5 \times 10^{3} \mathrm{~J} \cdot \mathrm{cm}^{-2}$. The mass absorption coefficient for carbon at $6.07 \AA$ is about $3.3 \times 10^{2} \mathrm{~cm}^{2} \cdot \mathrm{g}^{-1}$. For a thin foil, this corresponds to $224 \mathrm{eV}$ absorbed by each atom. When the electrons come into equilibrium, assuming no energy has been transferred to the ions, the energy of an atom is given by

$$
E=\sum_{z=1}^{z} n_{z}(T) \varepsilon_{z}+z n_{z}(T) k T
$$

where $n_{z}(T)$ is the probability $y^{8}$ that the atom is in the $z^{\text {th }}$ ionization 9 state, $\varepsilon_{z}$ is the total ionization energy of the $z^{\text {th }}$ state, and $k$ is Boltzmann's constant. The solution of Eq. (4) for $\mathrm{E}=224 \mathrm{eV}$ is $\mathrm{T}=1.5 \times 10^{5}{ }^{\circ} \mathrm{K} \simeq 13 \mathrm{eV}$. About $95 \%$ of the carbon is 4 times ionized, almost all of the rest is 3 times ionized. For a carbon foil of density $2 \mathrm{~g} \cdot \mathrm{cm}^{-3}$, the electron density is $4 \times 10^{23} \mathrm{~cm}^{-3}$. Using $\ln \Lambda \simeq 8$ in Eq. (3), we find that $t_{c} \simeq 5 \times 10^{-3} \mathrm{fs}$, so the 
electrons are well described by a thermal distribution throughout the pulse of $\sim 100 \mathrm{fs}$ duration.

Transfer of energy from the electrons to the ions, which is necessary to produce bulk fluid motion, is much faster than the time scale of the laser pulse. If we assume that both the ions and electrons are in a Maxwellian distribution, then the cooling of the electrons is described by ${ }^{6}$

$$
\frac{d T}{d t}=\frac{T_{i}-T}{t_{e q}}
$$

where $T_{i}$ is the ion temperature and

$$
t_{e q}=\frac{3 m m_{i} k^{3 / 2}}{8(2 \pi)^{1 / 2} n_{i} Z_{i}^{2} e^{4} \ln \Lambda}\left(\frac{T}{m}+\frac{T_{i}}{m_{i}}\right)^{3 / 2} .
$$

In our case, where the ions are mostly 4 times ionized carbon, this becomes

$$
t_{e q}=2.4 \times 10^{-22} T^{3 / 2} \approx 1.4 \times 10^{-14} \mathrm{~s} .
$$

Hence, the ion temperature and electron temperature remain nearly the same throughout the laser pulse.

To give an upper bound on the motion of the tag atom, we take it to have a kinetic energy equal to the average ion temperature. Because the ion temperature follows the electron temperature very closely, the equilibrium temperature can be found from Eq. (4) by 
adding the term $3 / 2 n_{z}(T) k T$. We then find the final ion temperature to be $T_{i} \simeq 11 \mathrm{eV}$. The ion temperature is zero at the beginning of the pulse and $11 \mathrm{eV}$ at the end of the pulse, so the average ion temperature is $\sim 5.5 \mathrm{eV}$. The average speed of a bromine tag atom is, therefore, about $3.5 \times 10^{5} \mathrm{~cm} \cdot \mathrm{s}^{-1}$, so it moves at most about $3.5 \AA$ during the pulse.

The local sound speed is a better estimate of motion of the outer edge of the foil and, to the extent that the expansion can be described by fluid dynamics, a similarity-flow description would give the majority of the fluid a much lower speed than the edge. Therefore, the actual motion would be expected to be considerably less than $3.5 \AA$. Finally, because the motion will be primarily in the longitudinal direction, and our measurements are confined to the transverse plane, we expect blurring to be negligible.

c. Influence of Reference Scattering Sphere on Image Quality

Movement of the reflecting surface of the reference scattering sphere will slightly shift the phase of the reference wavefront. This phase-shift can distort the holographic pattern of interference. The usable reflecting surface is between $6^{\circ}$ and $13^{\circ}$, with an average angle of about $9^{\circ}$. The effective intensity is thereby reduced multiplicatively by a factor of 0.16. Because it has a large atomic weight and large mass absorption coefficient, we cannot use tungsten as a reference scatterer with an $\mathbf{x}$-ray laser light source. Nickel is only a factor of two less reflective, and will suffer far less motion during the pulse. The mass absorption coefficient for nickel at $6.03 \AA$ is about $1.8 \times 10^{3} \mathrm{~cm}^{2} \cdot \mathrm{g}^{-1}$. The energy deposited in a thin layer at $9^{\circ}$ is, therefore, about $950 \mathrm{eV}$ per atom. From Eq. (4), this corresponds to a temperature of about $3.5 \times 10^{5}{ }^{\circ} \mathrm{K}$ or about $30 \mathrm{eV}$. Correspondingly, the surface moves about $4 \AA$ during the 100 fs pulse. 
The phase shift at the recording surface, at an angle $\theta$ from the laser axis, is given by

$$
\phi \approx \frac{2 \pi \Delta r \sin \theta}{\lambda}
$$

where $\Delta \mathrm{r}$ is the change in the reference-scattering-sphere radius. Taking $\theta=18^{\circ}$, we find $\phi$ $\simeq 74^{\circ}$, which would be acceptable. Again, this is an upper bound to the motion and the real change in radius could be substantially less.

\section{CONCLUSIONS}

Direct holographic imaging of biological materials is widely applicable to the study of the structure, properties and action of genetic material. A particular application involves the sequencing of the human genome. This new genomic imaging technology is composed of three subtechnologies. They are (1) an x-ray holographic camera, (2) suitable chemistry and enzymology for the preparation of tagged DNA, and (3) the illuminator in the form of an $\mathrm{x}$ ray laser.

The results of the research conducted under the support of this grant lead to three general conclusions concerning this emerging technology. They are $(\alpha)$ that an appropriate $\mathrm{x}$-ray camera, embodied by the instrument developed by MCR, is available and $(\beta)$ that suitable chemical and enzymatic procedures exist for the preparation of the necessary tagged DNA strands. The third conclusion $(\gamma)$ concerns the future development of the $x$-ray illuminator. The finding here, which is anchored on the results of research in several areas, stemming directly from work conducted by the principals of MCR, is that a practical small scale $\mathrm{x}$-ray light source is indeed feasible. This outcome requires the use of unconventional 
physical processes in order to achieve the necessary power-compression in the amplifying medium. The understanding of these new physical mechanisms is developing rapidly. Importantly, although the $\mathrm{x}$-ray source does not currently exist, the research has established the basic scaling laws that will determine its properties. When this $\mathrm{x}$-ray source becomes available, an extremely rapid and cost effective instrument for 3-D imaging of biological materials can be applied to a wide range of biological structural assays, including many questions regarding the sequencing and organization of the human genome. 


\section{REFERENCES}

1. W.S. Haddad, D. Cullen, J.C. Solem, J.W. Longworth, A. McPherson, K. Boyer, and C.K. Rhodes, "Fourier-Transform Holographic Microscope", Appl. Opt. 31 , 4973 (1992).

2. T. Gantier, M. Robert-Nicoud, M-N. Guilly, and D. Hernandez-Verdum, "Relocation of Nucleolar Proteins around Chromosomes at Mitosis", J. Cell Sci. 102, 729 (1992).

3. A.B. Borisov, A.V. Borovskiy, V.V. Korobkin, A.M. Prokhorov, O.B. Shiryaev, X.M. Shi, T.S. Luk, A. McPherson, J.C. Solem, K. Boyer, and C.K. Rhodes, "Observation of Relativistic and Charge-Displacement Self-Channeling of Intense Subpicosecond Ultraviolet (248 nm) Radiation in Plasmas", Phys. Rev. Lett. 68, 2309 (1992).

4. A.B. Borisov, A.V. Borovskiy, O.B. Shiryaev, V.V. Korobkin, A.M. Prokhorov, J.C. Solem, T.S. Luk, K. Boyer, and C.K. Rhodes, "Relativistic and ChargeDisplacement Self-Channeling of Intense Ultrashort Laser Pulses in Plasmas", Phys. Rev. A 45, 5830 (1992).

5. W. Bambynek, B. Crasemann, R. W. Fink, H. U. Freund, H. Mark, C. D. Swift, R. E. Price, P. Venugopala Rao, "X-Ray Fluorescence Yields, Auger, and CosterKronig Transition Probabilities", Rev. Mod. Phys. 44, 718 (1972).

6. L. Spitzer, Physics of Fully Ionized Gases, Interscience Publishers, NY (1962).

7. E. Plechaty, et al., LLNL Report UCRL-50400, Vol. 6, Rev. 3; J. Kirz and H. Rarback, "Soft X-Ray Microscopes", Rev. Sci. Instrum. 56, 1 (1985).

8. J. Michael Shull and M. Steenberg, "The Ionization Equilibrium of Astrophysically Abundant Elements", Astrophys. J. Supplement. Series 48, 95 (1982).

9. T. Carlson, et al., Atomic Data 2, 63 (1970); C. Moore, Atomic Energy Levels, NSRDS-NBS 35, (USGDO, WDC, 1971). 
FINAL TECHNICAL REPORT

Subcontract No. 090189-DoE-1 under DoE Prime Contract No. DE-FG0289ER60898

\title{
HUMAN GENOME SEQLENCING WITH DIRECT X-RAY HOLOGRAPHIC IMAGING
}

\author{
PERIOD \\ FEBRUARY, 1991 TO FEBRUARY, 1992
}

\author{
ROBERT M. MORIARTY \\ STEROIDS, LTD. \\ CHICAGO TECHNOLOGY PARK \\ CHICAGO, ILLINOIS
}




\section{TABLE OF CONTENT}

Page

I. Oligonucleotide Synthesis 3

II. Side-Chain Modification of Bases 3

Scheme I: $\quad 3$

III. Incorporation of 5-iodo-2'-deoxycytidine-5' triphosphate and 5- 4 bromo-2'-deoxyuridine 5-triphosphate by a polymerase.

IV. Metalation of DNA 4

$\begin{array}{ll}\text { Scheme II } & 5\end{array}$

V. Equipment on Hand 6

$\begin{array}{ll}\text { VI. Final Invoice } & 7\end{array}$ 


\section{Oligonucleotide Synthesis}

5-Bromo-2'-deoxyuridine 5-triphosphate was used as a base to synthesize a 10-mer using an automated method with the ABI-Model 392.

Specifically 5-bromo-2'-deoxyuridine was attached to the column at the $3^{\prime}$ end and subjected to a cycle with 5-bromo-2'-deoxyuridine-5'-OTMD-3'-N-de(isopropyl) methoxyphosphoramidate. Coupling with tetrazole catalyst, $\mathrm{I}_{2}$-morpholine oxidation, $\mathrm{Ac}_{2} \mathrm{O}$ capping, and $\mathrm{CF}_{3} \mathrm{COO} \mathrm{C}$ detritylation yielded the dimer. Repetition yielded ultimjatelya 12 -mer which was assayed by HPLC to $\sim 80 \%$ pure. The 10 -mer was $98 \%$ pure (HPLC).

\section{Side-Chain Modification of Bases}

Compounds , $\underline{4 a-c}$ and $\underline{5-c}-\mathrm{c}$ in Scheme I represent a new class of analogs which are of key importance for the type of process needed in our sequencing method. The vinyl double bond in $\underline{4 a-c}$ and allylic double bond is $\underline{5 a-c}$ serve as centers for further halogeneation.

\section{Scheme I:}

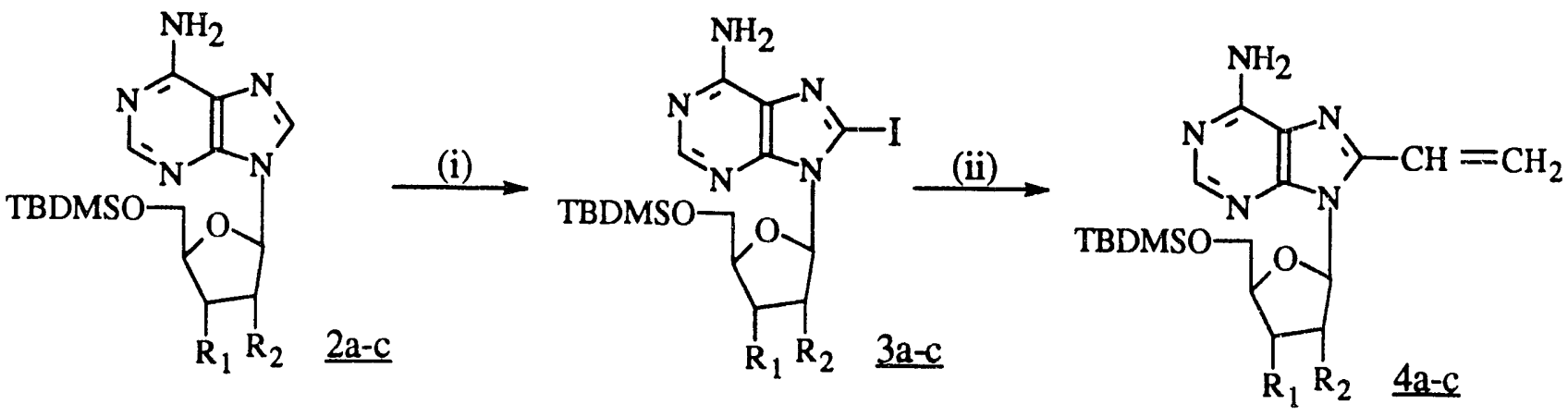

$2 \mathrm{a}-5 \mathrm{a}-\mathrm{R}_{1}, \mathrm{R}_{2}=$ OTBDMS

$2 \mathrm{~b}-5 \mathrm{~b}-\mathrm{R}_{1}, \quad=$ OTBDMS, $\mathrm{R}_{2}=\mathrm{H}$

$2 c-5 c-R_{1}, R_{2}+H$ (iii)

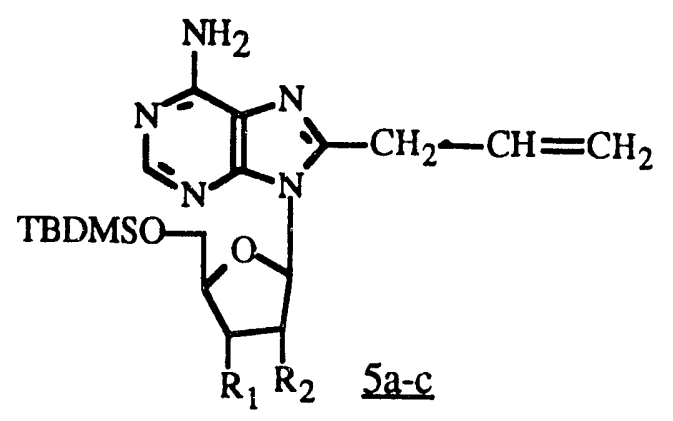


Using the following conditions:

i) LDA, $-78^{\circ} \mathrm{C}$, THF, 12 (ii)vinyltributyltin, $\mathrm{PD}\left(\mathrm{PPh}_{3}\right)_{4}, \mathrm{DMF}$, r.t. to $95^{\circ} \mathrm{C}$ (iii allyltribylyltin, $\mathrm{Pd}\left(\mathrm{PPh}_{3}\right)_{4}$, HMPA, r.t. to $145^{\circ} \mathrm{C}$.

We have scaled up the synthesis of $4 \mathrm{a}-\mathrm{c}$ and $5 \mathrm{a}-\mathrm{c}$. It is also important to note that these compounds are formed in the dideoxy modification (potential terminators) as well as deoxy and ribose (diol) types. The allylation has been extended to 5-iodo-2'-deoxycytidine and 5-iodo-2'-deoxyuridine.

\section{Incorporation of 5-iodo-2'-deoxycytidine-5' triphosphate and 5- bromo-2'-deoxyuridine 5-triphosphate by a_polymerase.}

The above two compounds were synthesized and given to Richard Baldarelli for incorporationIn a letter of October 1, 1991 to James Longworth details of the successful incorporation of these two bases are described.

\section{Metalation of DNA}

We have succeeded in conjugating ethylenediammine tetraacetic acid to the 3'-OH group of cytidine and adnosine. Both chelator nucleotides form a 1:1 complex with terbium (III) (Scheme II): 


\section{Scheme II}<smiles>CC1C2OC(n3cnc4c(N)ncnc43)C1OC(COP(=O)(O)O)C2O</smiles>

$\underset{\text { (2) } \mathrm{TbCl}_{3}}{\stackrel{\text { EDTA }}{\longrightarrow}}$

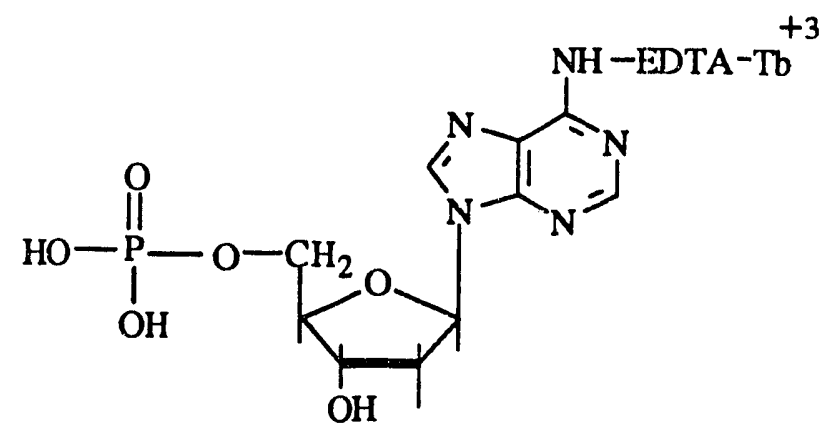

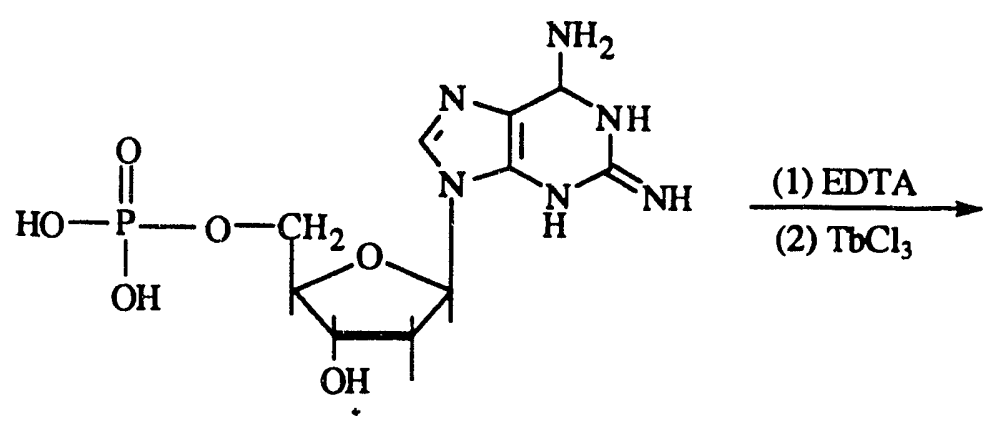

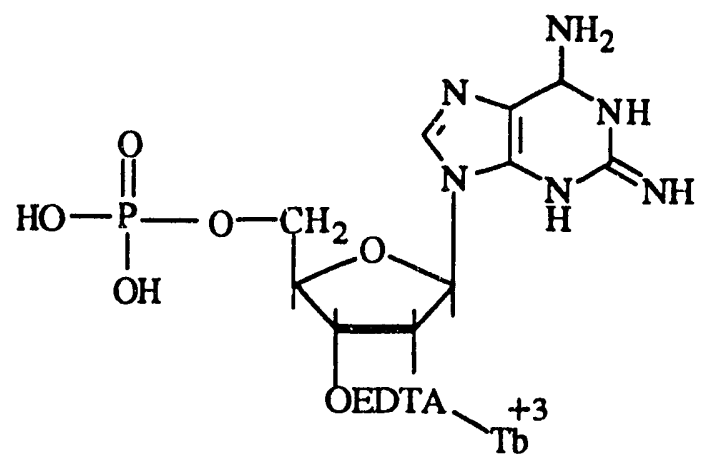<smiles>CC1C2OC(n3cnc4c(N)ncnc43)C1OC(COP(=O)(O)O)C2O</smiles><smiles>CCCCCCCCC(C)(C)C</smiles><smiles>[CH+][R17]([Pb])OC1C(COP(=O)(O)O)CC2(n3cnc4c(N)ncnc43)OC12</smiles> 


\section{Equipment on Hand}

An applied Bioscience Model 392 DNA synthesizer purchazed under this contract is housed Steroids, Ltd. 
October 1,1991

Dr. Jantes Longworth

MCR Technology Corp.

P.O. Box 10084

Chicago, II 60610-0084

Dear Jim,

I finally got around to that incoiporation experiment we cliscussed on the phone earlier. As I mentioned, I did some sequencing reactions with the halogenated nucleotides that your group sent (5-iodo-2'-

deoxycitidine-5' triphosphate (IC) and 5-bromo-2'-deoxyuridine-5' triphosphate (BU)). These experiments suggested that both modified nucleotides are incorporated by sequenase. There was a problem, however, in that not all of the termination reactions worked for all of the templates. Since each termination reaction was made up separately, the most likely explanation is that some of the termination mixes were prepared incorrectly.

I decided to test for elongation in a different way, one which would address elongation over a greater distance than could be addressed by sequencing alone. In this method, a primer bound to single-stranded M13 DNA was elongated in the presence of the different nucleotide combinations (i.e. four separate reactions: 1) rormal dNTPs, 2) IC instead of dCTP, 3) BU instead of dTTP and 4) IC and BU instead of dCTP and dTTP respectively). The polymerase used in this case was the Klenow fragment of DNA polymerase I. Elongation products of each of the four reactions were cut with a series of restriction enzymes. Regions of the M13 template passed by the elongating polymerase would be double-stranded and thus serve as suitable substrates for restriction enzymes. Regions of M13 DNA that were not reached by the polymerase would remain single-stranded and would not be cut by restriction enzymes.

Equal portions of each elongation reaction were digested with the following enzyme pairs: 1) AvalI + NaeI, 2) Avall + AlwNI, 3) Avall + BglI and 4) Avall + EcoRI. Avall has a single site in M13 at a position $290 \mathrm{bp}$ away from the primer binding site (in the direction of elongation) (see Fig. 1). The second enzymes of each pair also cut only once in M13 at positions of increasing distance away from the primer (see Fig. 1). The presence of a restriction fragment of predicted size (based on the distance between the Avall site and the site of the second enzyme used) indicates that polymerase elongation reached at least the site for the second enzyme.

The results indicate that for the majority of primed templates in each substitution reaction, the polymerase made it around the entire molecule ( 7250 bases). This indicates efficient incorporation of both $\mathrm{IC}$ and $\mathrm{BU}$, even when they are in the same reaction. There is, however, a noticeable difference in incorporation efficiency between IC and BU. The presence of incomplete elongation products (lighter bands of lower molecular weight) in the IC and IC + BU reaction lanes compared to the normal and BU reaction lanes (see fig. 2) indicates that IC is incorporaced less exífienty bain is BU. Elongation with the two substituted nucleotides $(I C+B U)$ was noticeably less efficient than with IC alone, but even in these cases, the majority of templates were converted to full length double-stranded molecules as evidenced by the intensity of higher molecular weight bands in these lanes. In truth, it is more appropriate to refer to these higher molecular weight bands as lower mobility bands, for the modified nucleotides may affect migration through the agarose gel in a manner that is, at least to some extent, molecular weight independent.

An additional result from this experiment is that when incorporated into DS.DNA, the two modified nucleotides are quite different with respect to their influence on the ability of restriction enzymes to cut this DNA. BU substituted DNA is digested nearly as well as is normal DNA, whereas, IC substituted DNA is digested very poorly. In fach, only the Avall + EcoRI reactions show any restriction products from IC substituted DNA.

In summary, this experiment indicates that both IC and BU can be incorporated by DNA polymerase in reactions that extend for at least $7250 \mathrm{bp}$. The amount of full length elongation products produced with the two modified nuclentides suggests that efficient incorporation could proceed on a significantly longer template. This is very encouraging for the future aimis of the project. 
Fig. 1

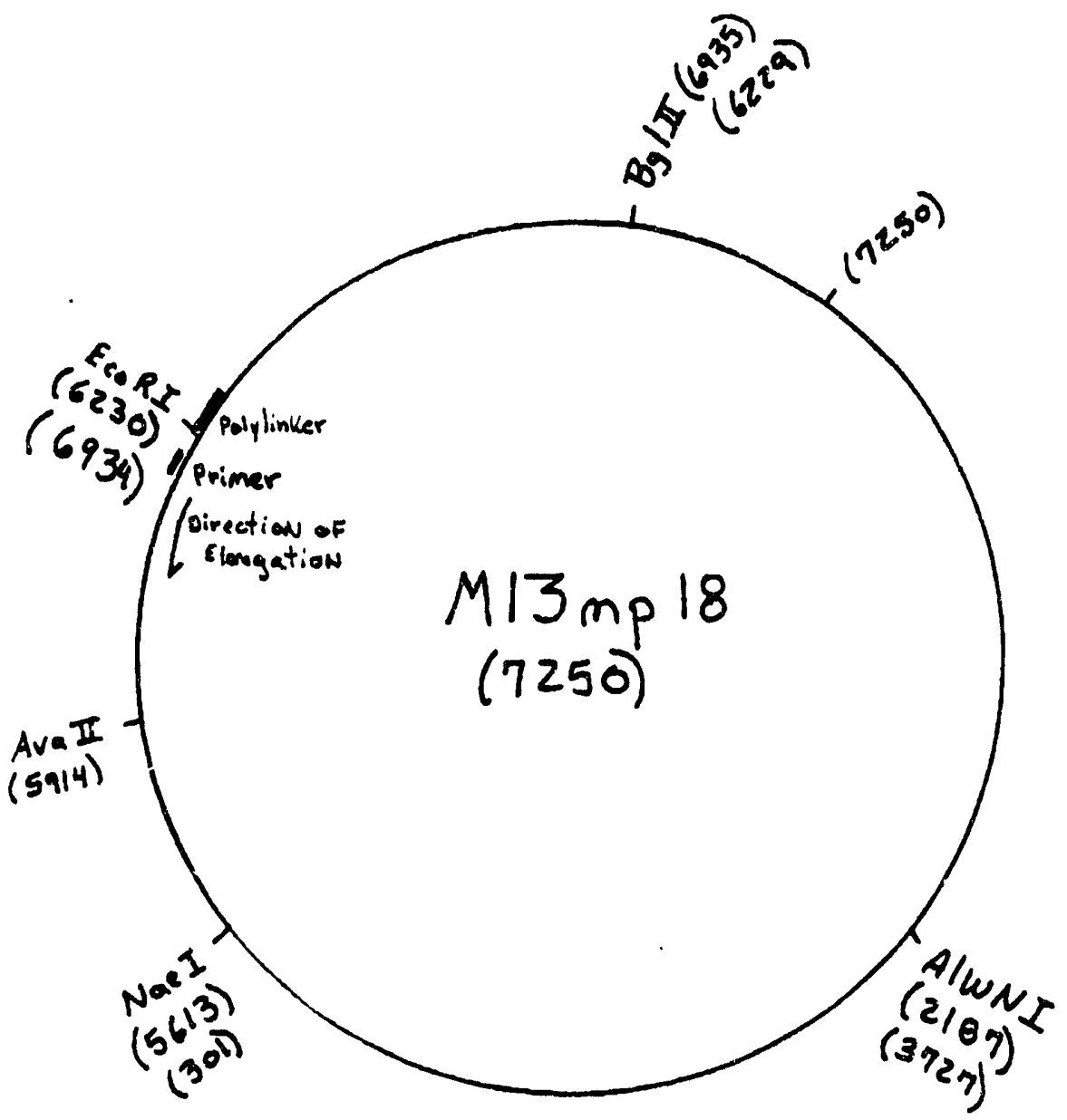

A) Map of M13mp18 with relevant restriction sites and predicted fragments.

B) Chart indicating restriction products expected frem full length elongation products or from RF (double-stranded) M13 DNA (in bp).

$\begin{array}{cccc}\text { AvalI + NaeI } & \text { AvalI+ AlwNI } & \text { Avall +BglII } & \text { AvalI + EcoRI } \\ 6949 & 3727 & 6229 & 6934 \\ 301 & 3523 & 1021 & 316\end{array}$

C) Restriction sites for the enzymes used.

$\begin{array}{ll}\text { AvalI } & \text { GGA/ICC } \\ \text { NaeI } & \text { GCCGGC } \\ \text { AIwNI } & \text { CAGNNNCTG } \\ \text { BglII } & \text { AGATCI } \\ \text { EcoRI } & \text { GAATIC }\end{array}$


Fig.1. Map of M13mp18 with relevant restriction sites and predicted fragments.

A) The black circle represents SS M13 DNA with restriction enzyme positions indicated where they would cut in DS DNA. Black numbers in parentheses represent enzyme site positions in M13mp18 based on the numbering system used by the NEB catalogue. The position of nucleotide 7250 (the arbitrarily defined beginning/end of sequence numbering) is also indicated. Numbers in red represent the distance (in the direction of elongation) between the Avall site and the respective second enzymes indicated. The sites of the primer (in blue) (the M13 hybridization probe primer was used) and the polylinker (in green) are shown. The distance between the primer and the polylinker is 26 bases.

B) A chart indicating restriction products expected from full length elongation products or from $R F$ (double-stranded) M13 DNA. One of the fragment sizes in each column corresponds to the red numbers in A. These represent the distances from the Avall site to the respective second enzyme sites. The other fragment size given in each column represents the distance from the respective second enzyme site to the Avall site continuing in the direction of elongation. The area between the primer and the end of elongation will remain single-stranded if elongation is incomplete. In the case of complete elongation, as appears to have occurred in most cases here, this area will simply be a nick in the polymerized strand adjacent to the primer.

C) Restriction sites for the enzymes used. Cytocines are indicated in green, Thymines in blue to highlight the positions in these sites where substitutions take place. 
Fig. $\dot{L}$
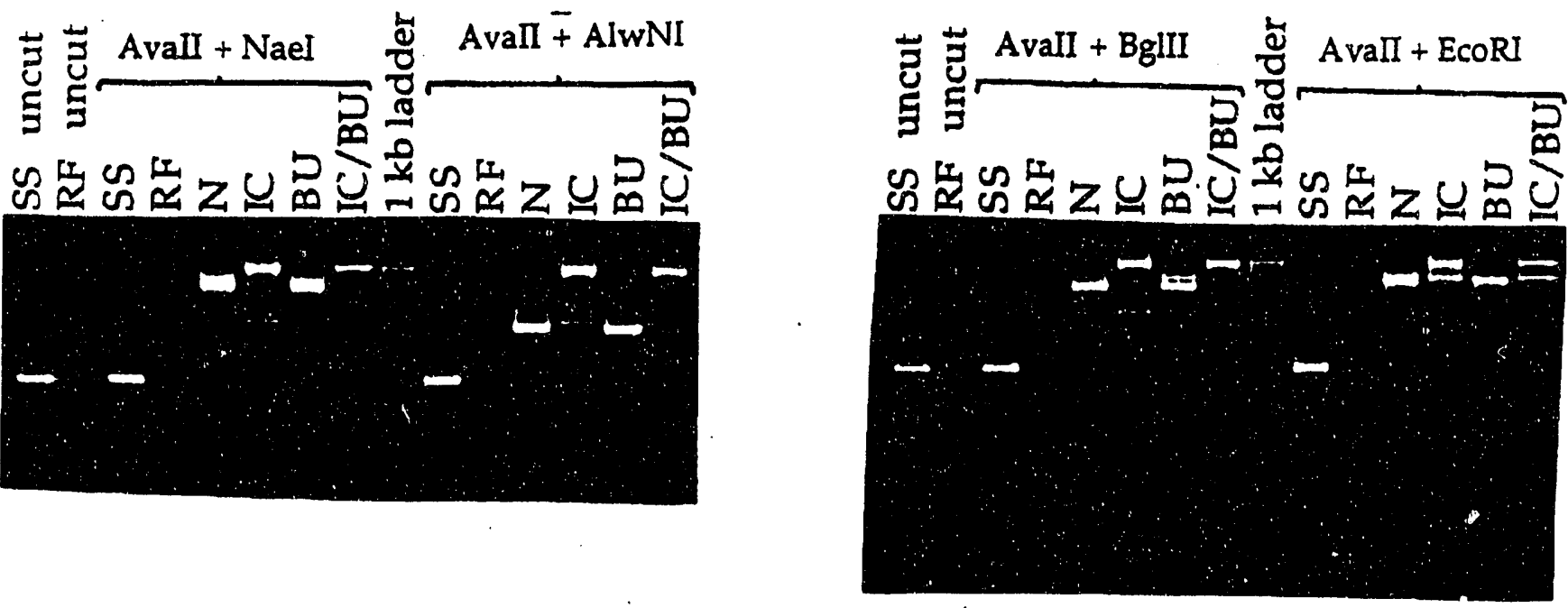
Fig.2. Agarose gels showing restriction digested elongation products.

Size fractionation of elongation products on $1 \%$ agarose gels after digestion with the restriction enzymes indicated is shown; $S S=$ single-stranded $M 13 \mathrm{mp} 18$ template DNA, RF= replicative form (double-stranded) M13mp18 DNA, N= elongation with normal nucleotides, IC= elongation with IC substituting for dCTP, $\mathrm{BU}=$ elongation with $\mathrm{BU}$ substituting for $\mathrm{DTTP}, \mathrm{IC} / \mathrm{BU}=$ elongation with $\mathrm{IC}$ and $\mathrm{BU}$ substituting for $\mathrm{dCTP}$ and dTIP respectively. The fragments of the $1 \mathrm{~kb}$ ladder marker lanes that are highlighted by dots have the following sizes: (from bottom to top) 1018 bp,2036 bp, 3054 bp, 4072 bp, 5090 bp, 6108 bp and 7126 bp.

\section{INTERPRETATIONS}

$$
\text { Avall }+ \text { NaeI }
$$

Unfortunately, the $301 \mathrm{bp}$ Avall-Nael band ran off the bottom of the gel, however, the presence of high molecular weight products from all elongation reactions suggests efficient incorporation for each. The reactions containing IC give rise to higher MW (lower mobility) products than do the reactions containing normal or BU nuclentides. This may be due to a combination of the higher MW and potential MW. independent mobility effects associated with the iodo group. Efficient incorporation is further demonstrated by the small amounts of single-stranded template remaining in the elongation reaction lanes (arrow). The amount of single-stranded template in the elongation reactions was equal to that loaded in the SS lanes on these gels, thus conversion to higher MW DNA is nearly complete in all elongation reactions.

\section{Avall + AlwNI}

The expected fragments produced by digestion with these enzymes ( $3727 \mathrm{bp}$ and $3523 \mathrm{bp}$ ) run as a close doublet. In the lanes containing digested elongation products from the normal nucleotide $(\mathbb{N})$ and $B U$ reactions, these two fragments run as a single band, though they are resolved into the two bands in the RF lane, which has much less DNA. The light bands in the IC and IC/BU lanes that run in approximately the some position as the expected doublet are probably incomplete elongation products, since these same bands appear in all of the IC and IC/BU lanes except for the ones with Avall + EcoRI products. Although IC appears to have interfered with the Avall + AlwNI digestion, again, the presence of high MW products at the expense of SS template indicates efficient incorporation.

$$
\text { AvalI + BglII }
$$

This set of reactions resembles the Avall + AlwNI reactions, for once again the $N$ and $B U$ lanes show the expected fragments ( $6229 \mathrm{bp}$ and $1021 \mathrm{bp}$ ), while the IC and IC/BU lanes show only high MW products. 
Avall +.EcoRI

Here, the expected fragments ( $6934 \mathrm{bp}$ and $316 \mathrm{bp}$ ) are observed in the $\mathrm{N}$ and $\mathrm{BU}$ lanes, but there also appears to be some 6934 bp product in the IC and IC/BU lanes (the 316 bp band would be too faint to detect, given the level of 6934 bp product in these lanes). Given the high MW products in the other IC and $\mathrm{IC} / \mathrm{BU}$ lanes as evidence of efficient elongation, this result for the AvaII + EcoRI digestion is more interesting in that these enzymes were able to cut IC substituted sites. However, this does demonstrate clearly that the polymerase was able to extent at least to the EcoRI site in the polylinker, which is almost entirely around the $\mathrm{M} 13$ vector from the primer binding site. 

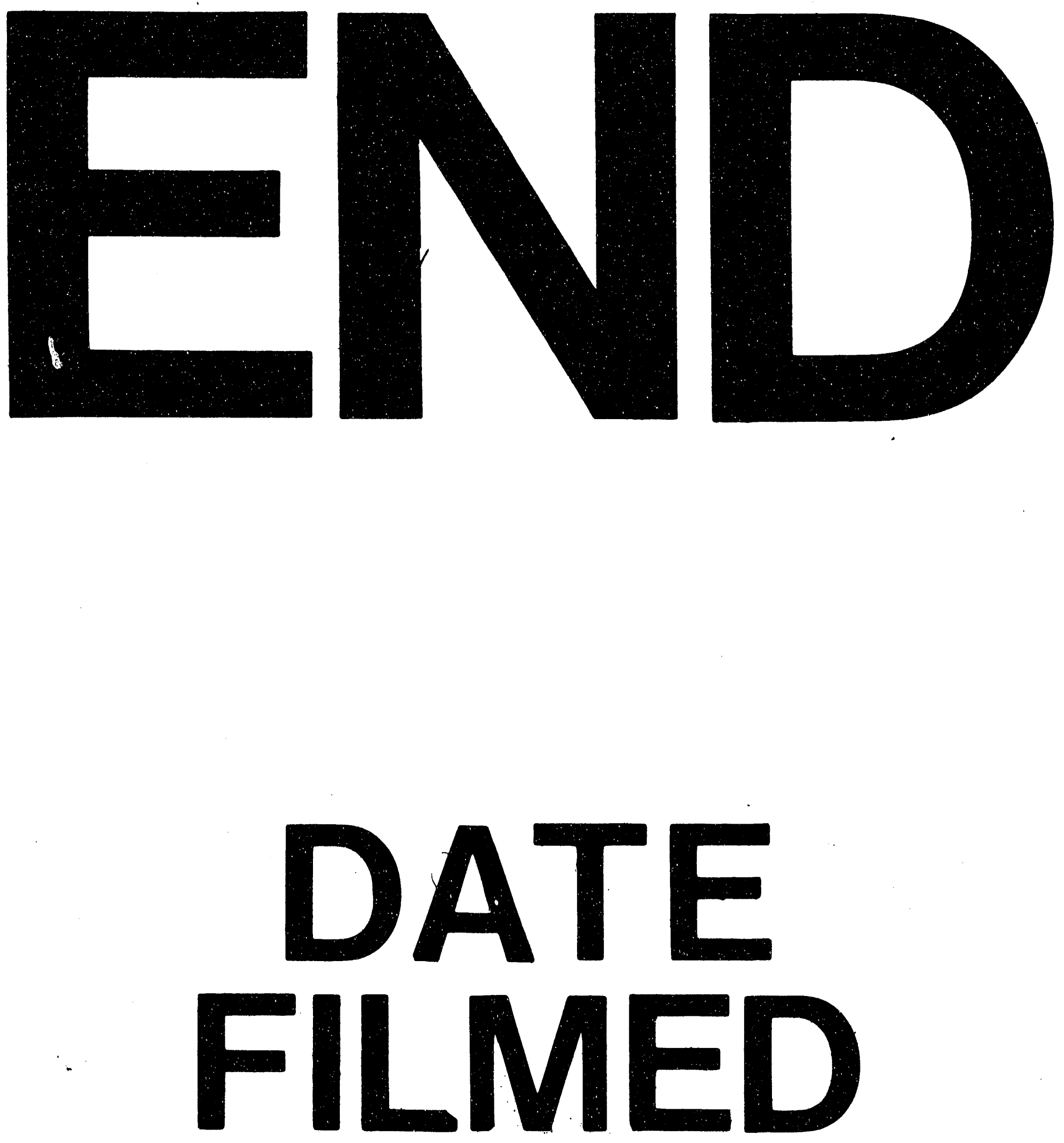

i

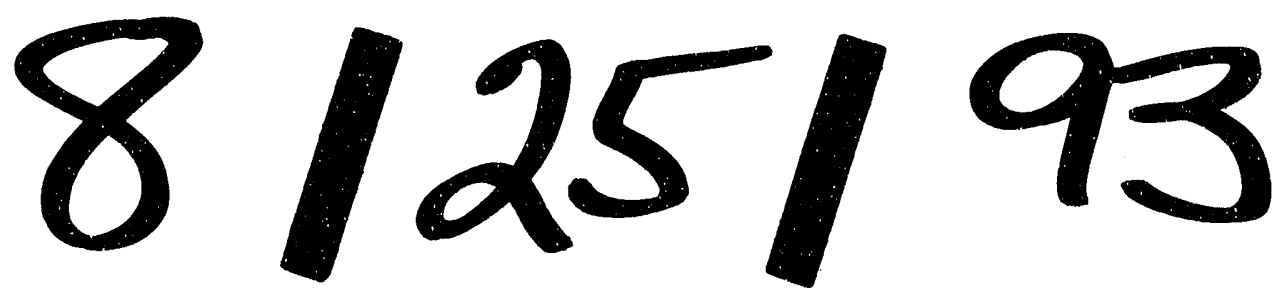


